\title{
Relationship Between the Anxious Symptoms and the Neurotransmitter in Parkinson's Mice with Different Dosages of MPTP
}

\author{
Yu Xia ${ }^{1 *}$, Suzhen $\mathrm{Ye}^{2}$, Jian $\mathrm{Shi}^{3}$, Hanjin Huang ${ }^{4}$ \\ ${ }^{1}$ Hospital of Wenzhou Medical University, Wenzhou, Zhejiang Province, China. ${ }^{2}$ Hospital of Wenzhou Medical \\ University - Department of Rehabilitation, Wenzhou, Zhejiang Province, China. ${ }^{3}$ Mianyang Central Hospital - \\ Department of Neurology (Psychosomatic Diseases), Mianyang Shi, Sichuan Sheng, China. ${ }^{4}$ Hospital of Wenzhou \\ Medical University - Department of Neurology, Wenzhou, Zhejiang Province, China
}

\begin{abstract}
This study aims to explore the relationship between the anxious symptoms and the impairment of 5hydroxytryptamine system in PD mice induced by different dosages of MPTP. The mice from the three model groups, the low-dose, dose and high-dose group, took longer time in the dark box than those in the control group $(P<0.05)$. However, no statistically significant differences were found among the model groups. The number of open arm entry $(O E)$ and the open arm time $(O T)$ were significant lower in the model group than those in the control group in the elevated plus-maze test $(P<0.05)$. The percentage of $O E$ in modle group was significantly lower compared with the control group $(P<0.05)$. The concentrations of striatum DA, HVA, 5-HT, and 5-HIAA were significantly reduced in the three model groups compared to the control group $(P<0.05)$. The 5-HT concentrations of high-dose group was significantly lower than those of the control group in the prefrontal cortex $(P<0.05)$. Anxiety symptoms were appeared in the three model groups of early PD mice, but no difference existed among these groups. The 5-hydroxytryptamine system was damaged after MPTP injection, which could lead to anxiety. However, the impairment of 5-hydroxytryptamine system induced by MPTP was dose-independent.
\end{abstract}

Keywords: Parkinson's disease; 1-methyl-4-phenyl-1,2,3,6-tetrahydroPyridine (MPTP); 5-hydroxytryptamine; Dopamine; Anxiety

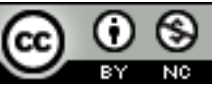

\footnotetext{
*Author for correspondence: medxiayu@ $163 . c 0 m$
} 


\section{INSTRUCTION}

Parkinson's disease (PD) is a typical motor disorder, which is accompanied by nonmotor symptoms such as anxiety, depression, cognitive impairment, and autonomic nervous system. In recent years, increasing epidemiological and clinical studies reported that non-motor symptoms appeared before the incidence of classical motor symptoms [1]. Besides motor disorder, $60-80 \%$ patients have various degrees of mental disturbances simultaneously, including depression, anxiety, sleep disturbances, dementia, and drug-induced mental disturbances. The influences of the mental disturbances on life quality of patients are more significant than the motor disorder [2]. It has been reported that the incidence rate of anxiety in PD patients reaches 39$60 \%$ [3]. Another study has also showed that more than $40 \%$ PD patients have generalized anxiety, fantod, and social phobia, and this rate was significantly higher than that in general populations [4]. Recently, increasing attentions have been paid on anxiety disorders in PD, which markedly affect the incidence, development, and prognosis of this disease. However, whether anxiety is a simultaneous phenomenon or a secondary psychosocial change of PD is still unclear. So far, most researches have focused on clinical observation of PD patients for anxiety disorders [5,6], but the evidence-based studies are still lacking. In this study, the anxious symptoms of acute PD mice induced by 1-methyl-4-phenyl-1,2,3,6-tetrahydropyridine (MPTP) were observed, and the relationship between anxiety and neurotransmitter and the influences of MPTP dosage on PD mouse model were discussed.

\section{MATERIALS AND METHODS}

\section{Animals and reagents}

Forty male C57BL/6J mice of 8 weeks old weighing 20-25 g were purchased from Shanghai Sliker Experimental Animal Limited Liability Company (CHN). The breeding was in clean level with circadian rhythms of $12 / 12 \mathrm{~h} \mathrm{light/dark} \mathrm{cycle,} \mathrm{and} \mathrm{the}$ animals got food and water adlibitum. The animal study was approved by the Institutional animal care and use committee of The First Affiliated Hospital of Wenzhou Medical University (permit No. PA2015347), and all animal procedures were performed according to the IACUC policy.

MPTP, standard materials of dopamine (DA), homovanillic acid (HVA), 5hydroxytryptamine (5-HT), and 5-hydroxyindoleacetic acid (5-HIAA) were purchased from Sigma company (USA), and the rest reagents were domestic analytically pure. The self-made elevated plus-maze, the light/dark box, and the High Performance Liquid Chromatography (HPLC) were from Agilent technology, Inc., and the refrigerated centrifuge was from Beckman Coulter, Inc. (USA).

\section{Grouping and modeling}

Forty C57BL/6 mice were randomly grouped into 4 groups by weight, and each group contained 10 mice which received continuously intraperitoneal injection for 8 days. MPTP $20 \mathrm{mg} / \mathrm{kg}$ for one day and normal saline (NS) $20 \mathrm{mg} / \mathrm{kg}$ for seven days were used for acute low-dose group (LG). MPTP $20 \mathrm{mg} / \mathrm{kg}$ and NS $20 \mathrm{mg} / \mathrm{kg}$ for four days were used for dose group (DG). MPTP $20 \mathrm{mg} / \mathrm{kg}$ for eight days was used for highdose group (HG). NS $20 \mathrm{mg} / \mathrm{kg}$ for eight days was used for the control group (CG).

\section{The light/dark box test}

Based on the test design of Crawley $J$ et al. [7], the light/dark box was $44 \mathrm{~cm}$ in length, $21 \mathrm{~cm}$ in width, and $21 \mathrm{~cm}$ in height. There were $2 / 3$ for light area and $1 / 3$ for dark area, and mice could pass the hole between these two areas. The incandescent lights were in top of light area. After the mice stayed in the box for $5 \mathrm{~min}$, the 
transition time between the two areas were countered by a counter, and the time in dark area was recorded by a timer.

\section{The elevated plus-maze test}

Based on the test design of Mudò [8], the maze was made of stainless steel, painted with matte black, and contained four arms. There was no wall for two open arms, but a $15.25 \mathrm{~cm}$-high wall on each side of the two closed arms. The arms were $5 \mathrm{~cm}$ in width, $30 \mathrm{~cm}$ in length, and attached to a solid metal stander which made the maze raising 40 $\mathrm{cm}$. The maze was put in a well-lighted room with six $32 \mathrm{~W}$ fluorescent lamps symmetrically set on top, and 2,800 flux was stably sustained by this illumination system. The mice were moved from the cages to the intersection of the open arms and the closed arms, and faced to the open arms direction and back to laboratory staffs, then movements of mice in the following 5 min were recorded as follows: (1) the number of open arm entry (OE) was counted when the mice entered any open arm with four paws, and one paw entirely leaving the arm was considered as a finish of one entry; (2) the time of staying in the open arm was recorded as open arm time (OT); (3) the number of close arm entry (CE) was counted when the mice entered any closed arm with four paws; (4) the time of staying in the closed arm was recorded as close arm time (CT). The percentage of OE and OT were calculated as $\mathrm{OE} \%=\%$ and $\mathrm{OT} \%=\%$, respectively.

\section{HPLC test}

The second day after the praxeology test, the mice received intraperitoneal anesthesia with $10 \%$ chloral hydrate $(4 \mathrm{ml} / \mathrm{kg})$, and the brain was taken to isolate the bilateral prefrontal cortex, hippocampus, and striatum, which were preserved in liquid nitrogen. The frozen tissues were weighed and $0.1 \mathrm{~mol} / \mathrm{L}$ perchloric acid was added $(1 \mathrm{mg}: 10$ $\mu \mathrm{l})$ and ultrasound homogenization was performed. The homogenate was centrifuged twice at $10,000 \mathrm{rpm} / \mathrm{min}$ at $4^{\circ} \mathrm{C}$, and the supernatant was filtered by microporous filter. The concentrations of DA, HVA, 5-HT, and 5-HIAA of the samples were analyzed with HPLC.

\section{Statistics}

The quantitative data was analyzed with SPSS 18.0 and expressed as $\square \mathrm{x} \square \mathrm{s}$. The data from four groups was analyzed by one-way analysis of variance (ANOVA). After pairwise comparison, lest significant difference (LSD) test was performed for data with equal variance and Dunnett' $\mathrm{T} 3$ test was performed for the rest data. $\mathrm{P}<0.05$ was considered statistically significant.

\section{RESULTS}

\section{The light/dark box test}

The results from the light/dark box test showed that the transition number of each group was different $(\mathrm{F}=3.084, \mathrm{P}<0.05)$, but no significant differences were found between the model group and CG or among the model groups. The CT differences of four groups were statistically significant $(\mathrm{F}=3.253, \mathrm{P}<0.05)$, so were the CT differences between the model group and $\mathrm{CG}(\mathrm{P}<0.05)$ (Table 1). However, the groups treated with different dosages of MPTP did not show significant differences in CT. The acute PD mice induced by MPTP stayed in the dark area for a longer time compared with the control mice, and showed corresponding anxious symptom, but there were no significant differences in time among the groups. 
Table 1. The transition number and CT of each group $(\mathrm{x} \pm \mathrm{s})$

\begin{tabular}{cccc}
\hline Group & $\begin{array}{c}\text { Mice } \\
\text { number }\end{array}$ & Transition number (count) & Closed arm time (second) \\
\hline CG & 10 & $8.8 \pm 1.8$ & $142.5 \pm 18.6$ \\
LG & 10 & $7.1 \pm 1.7$ & $167.1 \pm 24.0^{*}$ \\
DG & 10 & $8.6 \pm 1.9$ & $164.0 \pm 15.0^{*}$ \\
HG & 10 & $7.9 \pm 1.9$ & $170.8 \pm 28.9^{*}$ \\
\hline
\end{tabular}

Note : ${ }^{*}$ There were significant differences between each model group and $\mathrm{CG}(\mathrm{P}<0.05)$

The elevated plus-maze test

In the elevated plus-maze test, significant differences were found in OE, OT, OE\%, and OT\% in four groups $(\mathrm{P}<0.01)$. For OE, OT, and OT\%, the differences between the model group and CG were statistically significant $(\mathrm{P}<0.05)$, but no significant difference was found between any two model groups $(\mathrm{P}>0.05)$. Compared to $\mathrm{CG}$, the $\mathrm{OE} \%$ of either LG or DG showed significant differences $(\mathrm{P}<0.05)$. The OC\% difference between HG and CG was not statistically significant, and the OE\% difference among the model groups showed similar results $(\mathrm{P}>0.05)$ (Table2). The MPTP-induced acute PD mice stayed in open arms for a shorter time compared with the control mice, but the extent difference of anxiety caused by different dosages of MPTP was not statistically significant.

Table 2. The praxeology results of mice in elevated plus-maze test $(x \pm s)$

\begin{tabular}{cccccc}
\hline Group & $\begin{array}{c}\text { Mice } \\
\text { number }\end{array}$ & $\begin{array}{c}\text { Open arm entry } \\
\text { (OE) (count) }\end{array}$ & $\begin{array}{c}\text { Open arm time } \\
\text { (OT) }(\text { second) }\end{array}$ & $\begin{array}{c}\text { percentage of } \\
\text { OE }(\%)\end{array}$ & $\begin{array}{c}\text { percentage of } \\
\text { OT }(\%)\end{array}$ \\
\hline CG & 10 & $3.9 \pm 0.6$ & $34.9 \pm 11.9$ & $23.1 \pm 4.0$ & $12.9 \pm 4.9$ \\
LG & 10 & $2.0 \pm 0.8^{*}$ & $15.3 \pm 9.3^{*}$ & $14.4 \pm 5.0^{*}$ & $5.4 \pm 3.4^{*}$ \\
DG & 10 & $2.3 \pm 1.0^{*}$ & $14.5 \pm 9.5^{*}$ & $15.1 \pm 5.6^{*}$ & $7.9 \pm 3.4^{*}$ \\
HG & 10 & $2.9 \pm 1.1^{*}$ & $21.7 \pm 9.8^{*}$ & $19.6 \pm 5.8$ & $7.8 \pm 4.8^{*}$ \\
& & $\mathrm{~F}=6.724$ & $\mathrm{~F}=6.837$ & $\mathrm{~F}=5.038$ & $\mathrm{~F}=7.027$ \\
& & $\mathrm{P}=0.001$ & $\mathrm{P}=0.001$ & $\mathrm{P}=0.006$ & $\mathrm{P}=0.001$ \\
\hline
\end{tabular}

Note: $* \mathrm{P}<0.05$ was obtained by comparing with $\mathrm{CG}$

\section{Analyses of neurotransmitter in striatum by HPLC}

The concentrations of DA, HVA, 5-HT, and 5-HIAA in striatum were measured by HPLC, and the mean values of which were significantly different among four groups $(\mathrm{P}<0.05)$. For DA concentration, the differences between LG and CG, DG and LG, HG and DG were statistically significant $(\mathrm{P}<0.05)$. Comparing to $\mathrm{CG}$, the concentrations of HVA, 5-HT, and 5-HIAA showed obvious difference in the model groups, but there was no significant difference between any two model groups (Table 3 ). These results suggested that MPTP affected that DA in striatum of mice in a dosedependent manner, whereas MPTP influenced 5-HT concentration in striatum in a dose-independent manner. 
Table 3. The concentrations of neurotransmitters in striatum $(x \pm s)$

\begin{tabular}{cccccc}
\hline Group & $\begin{array}{c}\text { Mice } \\
\text { number }\end{array}$ & DA(ng/ml) & HVA(ng/ml) & 5-HT(ng/ml) & 5-HIAA(ng/ml) \\
\hline CG & 10 & $625.4 \pm 23.4$ & $121.0 \pm 31.7$ & $25.6 \pm 3.8$ & $27.4 \pm 5.1$ \\
LG & 10 & $455.7 \pm 58.1^{*}$ & $73.5 \pm 20.2^{*}$ & $19.3 \pm 3.3^{*}$ & $20.6 \pm 5.7^{*}$ \\
DG & 10 & $189.5 \pm 16.8^{*} \triangle$ & $54.9 \pm 7.7^{*}$ & $20.1 \pm 3.5^{*}$ & $21.4 \pm 2.7^{*}$ \\
LG & 10 & $138.5 \pm 13.2^{*} \triangle^{\#}$ & $43.4 \pm 8.4^{*}$ & $19.4 \pm 3.2^{*}$ & $21.9 \pm 3.7^{*}$ \\
& & $\mathrm{~F}=92.062$ & $\mathrm{~F}=24.184$ & $\mathrm{~F}=5.875$ & $\mathrm{~F}=3.992$ \\
& & $\mathrm{P}=0.000$ & $\mathrm{P}=0.000$ & $\mathrm{P}=0.034$ & $\mathrm{P}=0.019$ \\
\hline
\end{tabular}

Note: $* \mathrm{P}<0.05$ were respectively obtained by comparing with $\mathrm{CG}, \triangle \mathrm{P}<0.05$ were respectively obtained by comparing with $\mathrm{LG}, \# \mathrm{P}<0.05$ were respectively obtained by comparing with $\mathrm{DG}$

Analyses of neurotransmitter in hippocampus by HPLC

The differences of 5-HT and 5-HIAA concentrations between the model groups and CG were statistically significant $(\mathrm{P}<0.05)$, but the DA and HVA concentration in hippocampus of four groups were comparable $(\mathrm{P}>0.05)$. For the four neurotransmitters, the concentrations among the three model groups were not significantly different $(\mathrm{P}>0.05)$ (Table 4$)$. Our results suggested that, in hippocampus, MPTP could influence 5-HT but not DA.

Table 4. The concentrations of neurotransmitters in hippocampus $(\mathrm{x} \pm \mathrm{s})$

\begin{tabular}{llllll}
\hline \multicolumn{1}{c}{ Group } & $\begin{array}{c}\text { Mice } \\
\text { number }\end{array}$ & DA(ng/ml) & HVA(ng/ml) & $\begin{array}{c}5- \\
\text { HT(ng/ml) }\end{array}$ & $\begin{array}{c}5- \\
\text { HIAA(ng/ml) }\end{array}$ \\
\hline CG & 10 & $17.2 \pm 15.5$ & $5.5 \pm 5.1$ & $21.6 \pm 4.5$ & $22.5 \pm 3.1$ \\
LG & 10 & $15.9 \pm 14.7$ & $4.2 \pm 6.2$ & $11.1 \pm 2.8^{*}$ & $11.9 \pm 2.0^{*}$ \\
DG & 10 & $18.3 \pm 14.3$ & $4.4 \pm 2.9$ & $11.6 \pm 3.0^{*}$ & $13.4 \pm 3.5^{*}$ \\
HG & 10 & $15.9 \pm 9.1$ & $7.5 \pm 2.1$ & $14.3 \pm 5.5^{*}$ & $15.1 \pm 4.0^{*}$ \\
& & $\mathrm{~F}=0.057$ & $\mathrm{~F}=0.914$ & $\mathrm{~F}=14.307$ & $\mathrm{~F}=17.026$ \\
& & $\mathrm{P}=0.982$ & $\mathrm{P}=0.447$ & $\mathrm{P}=0.000$ & $\mathrm{P}=0.000$ \\
\hline
\end{tabular}

Note: * $\mathrm{P}<0.05$ was obtained by comparing with $\mathrm{CG}$

Analyses of neurotransmitter in cortex by HPLC

In cortex, the concentration differences of 5-HT were statistically significant among the four groups $(\mathrm{P}<0.05)$, but the concentrations of DA, HVA, and 5-HIAA showed no significant differences among these groups $(\mathrm{P}>0.05)$. Comparison of 5-HT concentrations between the model and control groups showed that differences between HG and CG, HG and DG were statistically significant (Table 5). These results suggested that MPTP potentially caused 5-HT damage in cortex. However, it was still unknown whether the extent of 5-HT damage was resulted from different dosages of MPTP. 
Table 5. The concentrations of neurotransmitters in cortex $(x \pm s)$

\begin{tabular}{cccccc}
\hline Group & $\begin{array}{c}\text { Mice } \\
\text { number }\end{array}$ & DA(ng/ml) & HVA(ng/ml) & 5-HT(ng/ml) & 5-HIAA(ng/ml) \\
\hline CG & 10 & $15.3 \pm 9.2$ & $9.8 \pm 4.6$ & $8.5 \pm 1.7$ & $8.9 \pm 1.9$ \\
LG & 10 & $9.3 \pm 3.5$ & $7.5 \pm 5.3$ & $6.7 \pm 2.0^{*}$ & $9.1 \pm 2.3$ \\
DG & 10 & $17.7 \pm 20.1$ & $13.3 \pm 14.4$ & $7.9 \pm 1.3$ & $9.3 \pm 2.5$ \\
LG & 10 & $12.1 \pm 7.2$ & $8.1 \pm 2.6$ & $5.8 \pm 1.2^{*}$ & $6.9 \pm 1.1$ \\
& & $\mathrm{~F}=0.709$ & $\mathrm{~F}=0.769$ & $\mathrm{~F}=4.858$ & $\mathrm{~F}=2.468$ \\
& & $\mathrm{P}=0.555$ & $\mathrm{P}=0.522$ & $\mathrm{P}=0.008$ & $\mathrm{P}=0.084$ \\
\hline
\end{tabular}

Note: $* \mathrm{P}<0.05$ was obtained by comparing to $\mathrm{CG}$

\section{DISCUSSION}

C57BL/6 mice are one of the most sensitive animals to MPTP, a common drug inducing PD. The MPTP-induced PD mice well reveals the changes of neuro pathogenesis and biochemistry in PD. It selectively damages the dopaminergic neurons in substantia nigra striatum system, which results in loss of DA and the appearance of selective motor disorders [9]. The PD mice induced by different dosages of MPTP would cause different changes of neuro biochemistry [10]. In this study, MPTP was used to induce acute PD mouse model. Our results showed that the concentrations of DA and HVA in striatum significantly decreased after MPTP injection, and the extent of DA damage was correlated with the dosage of MPTP as the high-dose of MPTP led to a greater reduction of DA (Table.3). It has been reported that acute MPTP modeling led to damage of intracerebral DA system [10,11]. When loss of DA nerve fibers in striatum is more than $70-80 \%$ or more than $50-60 \%$ of DA neurons in substantia nigra compactly died, patients would show clinical symptoms of PD [12]. Our results suggested that the PD models were induced successfully. However, the reduction of DA and HVA in hippocampus and cortex was no observed in our models, which was inconsistent with the previous reports. We speculated that this phenomenon might be due to the insufficient modeling time, which was not long enough to cause these damages in these areas.

Anxious disorder is a neurotic disorder with anxiety as the primary problem and mainly characterized by widespread and persistent anxiety and repeated attacks of panic. As a mental symptom, anxiety has both subjective and objective manifestations. The subjective expression is an anxiety mood that the patient is haunted with fear without a specific objective target and detailed perception. There are two aspects for objective expression, one is motor restlessness and muscle tension, and the other is dysfunction of autonomic nerve [13]. The elevated plus-maze and the light/dark box are the two widely accepted systems for testing mice anxiety [14,15]. The MPTPinduced PD mice showed early symptoms were used in the elevated plus-maze test and the light/dark box test in this study. In the light/dark box test, PD mice took a longer time in dark area, and in the maze test, mice of LG and DG took a shorter time in the open arms. These results suggested that PD mice showed obvious anxiety symptom at early time. Our results also showed that, compared to the control group, the transition number of PD mice between the light and dark areas was lower, while the total number of $\mathrm{OE}$ and $\mathrm{CE}$ had no significant differences, which would be explained by the symptom (acting slowly) of PD mice. Our results implied that anxiety was a simultaneous phenomenon of PD instead of the consequence of patient's psychological reactions. In the present study, we found that different dosages of MPTP did not induce different levels of anxiety of mice, which indicated that anxiety 
symptom was not only determined by MPTP, and the changes of intracephalic neurotransmitters in PD mice may also play a role in the development of anxiety.

It has been reported previously that about 50\% of PD patients had anxiety [16], and $40 \%$ of outpatients simultaneously had PD and anxiety disorder [17]. Anxiety disorder could not be ignored in treatment of PD patients. Dysthymic disorders, such as anxiety and depression, have been considered as the determinant factors of life quality of PD patients [18]. Interestingly, a retrospective cohort study with large sample population shows that a person having anxious personality is more easily to suffer from PD [19], which indicated an interrelationship between anxiety symptom and PD pathogenesis. A relationship between biochemistry change of non-DA neuron and anxiety in PD has been proposed, and this anxiety was considered to be organic [15]. Our results showed that the concentrations of 5-HT and 5-HIAA in the detected encephalic regions of MPTP-induced PD mice were decreased significantly compared with the control mice, consistent with the previous report by by Bower (2010) [9]. Rousselet et al. also found that acute MPTP modeling resulted in reduction of 5-HT in striatum and cortex of mice [20]. Vuckovic et al. using MPTP to induce acute mouse model and found that, 7 and 30 days after modeling, 5-HT concentration in prefrontal cortex in model mice significantly decreased compared to control mice [21]. It has been reported that defect of 5-HT transporter gene may increase the susceptibility to anxiety and depression of PD patients [22]. The 5-HT1A receptor is a self-receptor and regulates 5-HT release, antagonist of which leads to anxiety [23]. Using the 5-HT1A receptor antagonist WAY_100635 would reverse the antianxiety function induced by systemically or DRN locally using 5-HT1A receptor agonist 8-OH-DPAT. It was speculated that the antianxiety function of 8-OH-DPAT was achieved through activating 5-HT1A receptor of cytodendrite [24]. Our PD mice showed obvious anxiety symptoms, and HPLC detection of encephalic regions (prefrontal cortex, hippocampus, and striatum) found reduction of 5-HT and its metabolites. These data implied that the anxiety symptoms of MPTP-induced acute PD mice were related with encephalic 5-HT damage, and the system burdens appeared at early time of PD.

The concentration of DA and its metabolites HVA were greatly reduced in striatum but not in hippocampus and cortex, which differ from other previous report [25]. This difference might be explained by the modeling time that was not long enough to induce such damages in hippocampus and cortex. Our results implied that the anxiety of PD mice was not directly mediated by the DA mood regulation pathway that was in deutocerebrum limbic system and detocerebrum cortex system.

\section{CONCLUSION}

Our study showed MPTP induced anxiety and 5-HT damage in PD mice in a doseindependent manner. Further studies are needed to access whether the anxiety symptom and biochemistry change caused by subacute or chronic models were different from that caused by acute mouse model. How the anxiety disorder in PD patients is regulated by 5-HT is still needed further investigation.

\section{ACKNOWLEDGEMENTS}

We thank all the enrolled patients who support and contribute to this study. We thank Ling Zhang, Feng Qing, and Peiao Li for manuscript preparation and technique supports for this study. 


\section{REFERENCES}

1. Tolosa E, Compta Y, Gaig C The premotor phase of Parkinson's disease. Parkinsonism Relat Disord 13 (Suppl) 2007:S2-S7.

2. Gage H, Hendricks A, Zhang S, et a1. J Neurol Neurosurg Psychiatry, 2003,74:163.

3. Witjas T, Kaphan E, Azulay JP, et a1. Nonmotor fluctuations in Parkinson's disease : frequent and disabling[J]. Neurology, 2002, 59:108-413.

4. Richard IH. Anxiety disorders in Parkinson S disease. Adv Neurol. 2005, 96(1):42-55.

5. Rosa Quelhas MD, Manuela Costa MD. Anxiety, Depression, and Quality of Life in Parkinson's Disease[]]. The Journal of Neuropsychiatry and Clinical Neurosciences 2009; 21:413-419.

6. Liberatore GT, Jackson-Lewis V, Vukosavic S, et al. Inducible nitric oxide synthase stimulates dopaminergic neurodegeneration in the MPTP model of Parkinson disease[J]. Nature medicine, 1999, 5(12): 1403-1409.

7. Crawley J, Goodwin FK. Preliminary report of a simple animal behavior model for the anxiolytic effects of benzodiazepines. Pharmacol Biochem Behav. 1980, 13(2):167-70.

8. Mudò G, Mäkelä J, Di Liberto V, et al. Transgenic expression and activation of PGC-1 $\alpha$ protect dopaminergic neurons in the MPTP mouse model of Parkinson's disease[J]. Cellular and Molecular Life Sciences, 2012, 69(7): 1153-1165.

9. Cyr M, Beaulieu JM, Laakso A, et al. et al. Sustained elevation of extracellular dopamine causes motor dysfunction and selective degeneration of striatal GABAergic neurons. Proc Natl Acad Sci U S A. 2003,100(19):11035-40.

10. Noelker C, Morel L, Lescot T, et al. Toll like receptor 4 mediates cell death in a mouse MPTP model of Parkinson disease[J]. Scientific reports, 2013, 3.

11. Rollema H, Fries DS, de Vries JB, et al. HPLC-assay with electrochemical detection for the neurotoxin MPTP, its metabolite MPP+ and MPTP-analogues in biological samples after purification over Sephadex G10. Life Sci. 1985, 28;37(17):1633-40.

12. Nayyar T, Bubser M, Ferguson MC, et al. Cortical serotonin and norepinephrine denervation in parkinsonism: preferential loss of the beaded serotonin innervation. Eur $\mathbf{J}$ Neurosci. 2009, 30(2):207-16.

13. Volkers AC, Tulen JH, van den Broek WW, et al. Motor activity and autonomic cardiac functioning in major depressive disorder. J Affect Disord. 2003,76(1-3):23-30.

14. John f. Cryan, Andrew holmes. The ascent of mouse:advances in modelling human depression and anxiety. Nature reviews, drug discovery, september 2005(4),775-790

15. Lofrumento DD, Nicolardi G, Cianciulli A, et al. Neuroprotective effects of resveratrol in an MPTP mouse model of Parkinson's-like disease: possible role of SOCS-1 in reducing pro-inflammatory responses[J]. Innate immunity, 2014,20(3): 249-260.

16. Marinus J, Leentjens AF, Visser M, Stiggelbout AM, van HJJ. Evaluation of the hosPital anxiety and dePression scale in Patients with Parkinson's disease. Clin NeuroPharmacol. 2002. 25(6): 318-24.

17. Walsh K, Bennett G. Parkinson's disease and anxiety. Postgrad Med J 2001,77:89-93.

18. Carod-Artal FJ, Ziomkowski S, Mourao MH, Martinez-Martin P. Anxiety and dePression: main determinants of health-related quality of life in Brazilian Patients with Parkinson's disease. Parkinsonism Relat Disord. 2008, 14(2): 102-8.

19. Bower JH, Grossardt BR, Maraganore DM, et al. Anxious Personality Predicts an increased risk of Parkinson's disease. Mov Disord. 2010, 25(13): 2105-13.

20. Rousselet E, Joubert C, Callebert J, et al. Behavioral changes are not directly related to striatal monoamine levels, number of nigral neurons, or dose of parkinsonian toxin MPTP in mice. Neurobiol Dis. 2003,14(2):218-28.

21. Vuckovic MG, Wood RI, Holschneider DP, et al. Memory, mood, dopamine, and serotonin in the 1-methyl-4-phenyl-1,2,3,6-tetrahydropyridine-lesioned mouse model of basal ganglia injury. NeurobiolDis 2008,32(2):319-27.

22. Menza MA, Palermo B, DiPaola R, Sage JI, Ricketts MH. DePression and anxiety in Parkinson's disease: Possible effect of genetic variation in the serotonin transPorter. J Geriatr Psychiatry Neurol. 1999,12(2): 49-52.

23. Groenink L, Pattij T, De Jongh R, et al. 5-HT1A receptor knockout mice and mice overexpressing corticotropin-releasing hormone in models of anxiety. Eur J Pharmacol. 
2003, 28;463(1-3):185-97.

24. Park MH, Sanders E, Howe M, et al. Association of Anxiety Symptoms in Offspring of Bipolar Parents with Serotonin Transporter-Linked Polymorphic Region (5-HTTLPR) Genotype. J Child Adolesc Psychopharmacol. 2015, 25(6):458-66.

25. Lv H, Lin HY, Zhao H, et al. Effects of ningdong granule on DA, DRD2, and HVA in a rat model of Tourette's syndrome. J Tradit Chin Med. 2012, 32(2):283-8.

Received: August 25, 2016 Accepted: October 20, 2016 